ARTUR HAMRYSZCZAK - LUBLIN

\title{
BERICHT ÜBER DIE TAGUNG DIE ORGANISATION UND ARBEIT IN KIRCHLICHEN ARCHIVEN 24. SEPTEMBER 2007
}

Am 24. September 2007 fand im Hauptgebäude der Katholischen Universität Lublin „Johannes Paul II.” eine vom Institut für Kirchliche Archive, Bibliotheken und Museen (ABMK) organisierte wissenschaftliche Tagung unter dem Titel „Die Organisation und Arbeit in kirchlichen Archiven" statt.

Eröffnet wurde die Tagung vom Rektor der KUL P. Prof. Stanisłw Wilk. Eine Einführung in die Thematik des Symposiums gab der Direktor des ABMK-Instituts P. Prof. Anzelm Weiss.

In den von ihnen vorbereiteten Beiträgen behandelten die Redner verschiedene Probleme im Zusammenhang mit der Archivarbeit.

Hanna Krajewska hielt das Referat „Die Bearbeitung von Hinterlassenschaften im Archiv der Polnischen Akademie der Wissenschaften". In ihrem Beitrag verwies sie auf die Richtlinien für die Bearbeitung handschriftlicher Hinterlassenschaften, die von den Mitarbeitern der Akademie bei der Arbeit mit solchen Archivalien herangezogen werden. sie erinnerte außerdem an die Definition der Hinterlassenschaft als „Archivaliengruppe privater Herkunft” oder „,persönliches Archiv".Darüber hinaus zeigte sie, wie die Arbeit beim Ordnen von Hinterlassenschaften im Archiv der Polnischen Akademie der Wissenschaften in der Praxis aussieht.

Zum Thema der „Prophylaxe und Sicherung von Archivmaterial” sprach Piotr Dudzikowski. In seinem Beitrag erinnerte er an die wichtigsten Dinge, die bei der Arbeit mit Papierarchivalien zu beachten sind. Er erwähnte die physiko-chemischen Eigenschaften des Papiers, die Bedingungen seiner Aufbewahrung sowie verschiedene Faktoren, die die Zerstörung des Papiers bewirken können. Außerdem unterstrich er die Notwendigkeit der Prophylaxe, die beim Aufbewahren von Archivalien grundlegend ist. Danach präsentierte er die Konservierungstechniken, die angewandt werden, um der Zerstörung des Papiers vorzubeugen.

De nächste Beitrag stammte von P. W. Kujawski, der sich in seinem Referat „Die Rolle des Archivars beim Zusammentragen von Dokumentationen” mit den diesbezüglichen Aufgaben des Archivars befasste. Am Beispiel des Diözesanar- 
chivs in Włocławek demonstrierte er die Problematik der Archivarbeit. Er erinnerte an die Geschichte der kirchlichen Archive und der von ihnen aufbewahrten Bestände und machte deutlich, dass die in diesen Archiven gesammelten Archivdokumente zum nationalen Archivbestand gehören sowie dass die kirchlichen Archive, was die Technik des Sammelns der Bestände und die Sorge um ihre Sicherung betrifft, die Errungenschaften der staatlichen Archivistik und die von ihr erarbeiteten Methoden nutzen. W. Kujawski erinnerte auch an die Einteilung der kirchlichen Archive in Diözesan- und Ordensarchive und im Rahmen der Diözesanarchive jeweils in das Archiv der Diözesankurie, des Kirchengerichts sowie in die Pfarreiarchive.

P. M. Brudzisz präsentierte das Referat „Die Archivbestände der Polnischen Katholischen Missionen in Frankreich und in Deutschland sowie der Kongregation für außerordentliche kirchliche Angelegenheiten". In seinem Beitrag erinnerte er an die Geschichte der 1922 gegründeten Polnischen Katholischen Mission (PMK) in Paris. Danach verwies er auf den Charakter der von der Mission erstellten und gesammelten Archivalien: Briefe, Berichte, Akten von Seelsorgeeinrichtungen, religiösen Organisationen und zivilen polnischen Emigranten, Personalakten usw.

Darüber hinaus befasste sich M. Brudzisz mit der Polnischen Katholischen Mission in Deutschland, die für die aus den deutschen Konzentrationslagern befreiten Polen sowie die ehemaligen polnischen Zwangsarbeitern gegründet wurde. Diese Mission initiierte Papst Pius XII., indem er 1945 den Militärbischof Józef Gawlina zum Ordinarius der auf dem Territorium Deutschlands weilenden Polen ernannte. M. Brudzisz äußerte sich zum jetzigen Zustand des Archivs der Polnischen Katholischen Mission in Deutschland und stellte fest, dass es sich noch im Stadium der Inventarisierung befindet. Informationen über die Bestände und Hilfe bei ihrer Nutzung erteilen nicht nur provisorische Inventarverzeichnisse, sondern auch die Mitarbeiter der Mission.

Eine weitere Institution, deren Wirken P. Brudzisz in seinem Beitrag behandelte, war das 1793 gegründete Archiv der Kongregation für außerordentliche kirchliche Angelegenheiten. Die Bestände dieses Archivs umfassen zwei grundlegende Reihen: „Posizioni” und „Sessioni”, und darüber hinaus noch „Carte”. Die Problematik der katholischen Kirche im geteilten Polen tritt in allen Reihen in Erscheinung, und in der Reihe „Posizioni” geschieht dies in den Archivaliengruppen, die die Namen der Teilungsmächte tragen. Uns interessieren hier besonders die Gruppen „Russia - Polonia”, „Prussia - Polonia” und „Austria - Polonia”. 1905 entstand die Abteilung „Polonia”, die der kirchlichen Problematik vor allem in Kongresspolen gewidmet war. Eine wichtige Hilfe zur Erforschung des Inhalts dieser Reihen bietet ein über 30 Bände zählendes detailliertes Inventarverzeichnis. Eine wichtige von M. Brudzisz mitgeteilte Information betraf die Tatsache, dass die die Auslandspolen in Frankreich und in Deutschland betreffende Dokumentation im Archiv der Kongregation noch nicht vollständig inventarisiert ist. Der Referent erinnerte daran, dass das Ende des Pontifikats von Pius XI. (Anfang 1939) die Grenze bildet, bis zu der die Archivalien zugänglich sind. 
Das nächste Referat „Quellen zur Geschichte der katholischen Kirche in Wilnaer Archiven" hielt P. Adam Szot. Der Referent präsentierte die Geschichte des 1387 gegründeten Bistums Wilna. Danach befasste er sich mit dem Schicksal der von der bischöflichen Kurie, dem bischöflichen Gericht, d.h. dem Konsistorium, sowie dem an der Wilnaer Kathedrale existierenden Domkapitel erstellten Archivmaterialien. Pater Szot erinnerte daran, dass sich die aus der Diözese Wilna stammenden Pergamentdokumente in der Bibliothek der Litauischen Akademie der Wissenschaften in Vilnius befinden. In dieser Einrichtung werden auch die Materialien des Archivs des Wilnaer Domkapitels aufbewahrt. Nach der Verstaatlichung der kirchlichen Sammlungen waren auch die Bestände des Kurienarchivs in die Bibliothek der Litauischen Akademie der Wissenschaften übergegangen. Archivalien kirchlicher Herkunft befinden sich auch in der Bibliothek der Wilnaer Universität. Dabei handelt es sich um Materialien aus dem früheren bischöflichen Archiv, welches hauptsächlich die Visitationsakten enthält, die in der Zeit des 1. Weltkrieges nicht aus Wilna herausgeschafft wurden. In die Wilnaer Universitätsbibliothek wurden auch die Dokumente des Priesterseminars in Wilna verbracht.

Die meisten der von den kommunistischen Behörden konfiszierten Archivalien kirchlicher Herkunft gelangten in das Litauische Staatliche Historische Archiv in Vilnius (Materialien bis 1940) und ins Litauische Archiv Neuer Akten (Materialien nach 1940). Diese Archivalien umfassen u.a. Aktengruppen einzelner Pfarreien sowie Matrikelbücher. Zu den Beständen dieser Einrichtungen kamen auch die Archive anderer litauischer Diözesen (Żmudź und Sejny) hinzu. Die wertvollste Sammlung im Spezialarchiv der Litauischen Republik (Lietuvos Ypatingasis Archivas) bilden die Amtsakten des Bevollmächtigten für Kultfragen der Litauischen Sowjetrepublik, die Dokumente über die in Gefängnissen eingekerkerten oder Lager deportierten Priester sowie operatives Material über die Bespitzelung des Klerus enthalten.

P. Robert R. Kufel unternahm in seinem Referat „Der Stand der kirchlichen Archivistik in Polen" den Versuch einer Beurteilung der polnischen Kirchenarchivistik. In seinem Beitrag erinnerte er an die allgemeine Geschichte der kirchlichen Archivistik in Polen unter besonderer Berücksichtigung des 20. Jahrhunderts. Der Referent präsentierte das Lebensbild des hervorragenden Historikers und Archivars P. Dr. J. Kwolek, der 1918 von den Oberen der Diözese Przemyśl mit der Schaffung eines Archivs sowie mit der Sorge um die darin gesammelten Akten betraut wurde. R. Kufel verwies auf die Zwischenkriegszeit als bahnbrechend für die Entwicklung der kirchlichen Archivistik. Damals entstanden immer neue Diözesanarchive. Man begann die kirchlichen Sammlungen zu systematisieren und zu ordnen und gründete wissenschaftliche Einrichtungen.Der Ausbruch des 2. Weltkrieges hemmte die Entwicklung und die Entstehung weiterer Archivzentren. Die nicht wieder gutzumachenden Verluste an Archivbeständen gehen auf das Konto der Besatzer. Nach dem Krieg entstanden dann - trotz widriger Umstände - weitere Diözesanarchive. Sehr aktiv waren auf dem Gebiet der kirchlichen Archivistik damals P. Stanisław Librowski, P. Wincenty Urban und P. Hieronim Wyczawski OFM. 
Ein wichtiges Ereignis in der kirchlichen Archivistik nach 1945 war die Gründung des Zentrums Kirchlicher Archive, Bibliotheken und Museen (ABMK) an der Katholischen Universität Lublin im Jahre 1956. Es besaß den Status eines selbständigen wissenschaftlichen Instituts, das ab 1959 die Zeitschrift „Archiwa, Muzea i Biblioteki Kościelne" herausgibt.

Ein weiteres bahnbrechendes Ereignis für die kirchliche Archivistik bildete die Gründung der Vereinigung Kirchlicher Archivare (SAK) im Jahre 2004 in Warschau. Die Hauptaufgabe, die sich diese Vereinigung stellt, besteht im Ordnen, Zugänglichmachen und Inventarisieren kirchlicher Archivalien. Sie gibt regelmäßig ein Bulletin unter dem Namen „Archiva Ecclesiatica” heraus.

Nach den gehaltenen Referaten kam es zur Diskussion zwischen den Teilnehmern der Tagung zu den in den einzelnen Beiträgen behandelten Themen. 